\title{
Candida albicans Meningitis in an Infant with Noonan Syndrome
}

\author{
Faezeh Ahmadi ${ }^{1}$, Maedeh Ahmadi ${ }^{1}$, Bahareh Yaghmaei ${ }^{2}$, Farideh Nejat ${ }^{2}$, Nima Rezaei ${ }^{2,3}$ and Setareh Mamishi $^{1,2}$ \\ ${ }^{1}$ Infectious Disease Research Center, Tehran University of Medical Sciences; ${ }^{2}$ Department of Pediatrics, Pediatrics Center of Excellence, \\ Children's Medical Center, Tehran University of Medical Sciences; ${ }^{3}$ Growth and Development Research Center, Tehran University of Medical \\ Sciences; Tehran, Iran
}

\begin{abstract}
Noonan syndrome is a rare disorder, characterized by several malformations such as dysplasia and stenosis of the pulmonary valve, atrial septal defect and a typical pattern of hypertrophic cardiomyopathy. We describe here a 1month old girl, who was referred to our center with seizure and apnea. She had wide anterior fontanel, head circumference and sunset eye. Intaventricular hemorrhage by sonography and atrial septal defect and hypertrophy cardiomyopathy by echocardiography were detected. Clinical and laboratory findings of the patient were compatible with a diagnosis of Noonan syndrome, which was also confirmed by molecular analysis. Candida albicans was grown in the blood and cerebrospinal fluid cultures. Treatment with Amphotrycine B was started for the patient and she responded well to this therapy. Early diagnosis and appropriate diagnosis of a rare condition in the patient with such rare disease are the main keys to avoid further complications and even death of patient.
\end{abstract}

Key-Words: Candida barbicans meningitis, Noonan syndrome.

Noonan syndrome (OMIM\#163950) is a rare disorder, characterized by several malformations including craniofacial anomalies, such as ptosis, webbing of the neck and a deep nuchal hairline, as well as skeletal deformities such as short stature, clinodactyly, pectus carinatum and funnel chest and other organ anomalies, such as cardiac valve disease, testicular retention and kidney malformations [1]. This syndrome is a clinically homogeneous but genetically heterogeneous condition. Type 1 of this syndrome (NS1) is due to defined by the mutations in PTPN11 (OMIM*176876), a gene encoding the non-receptor protein tyrosine phosphatase SHP2, which is found in approximately $40 \%$ of the patients with Noonan syndrome [2].

Candida spp. meningitis is also a rare clinical situation; although it is recently becoming more frequent, especially in the cases with immunosuppressive conditions, such as drug addiction, malignancies, organ transplantation, and HIV infection [3]. Central nervous system (CNS) involvement could be occurring as a complication of neonatal candidiasis, which is a major challenge in establishing diagnosis and adequate treatment and can lead to neurodevelopment morbidity and death in a case of delay in diagnosis [4].

Here we describe an infant with Noonan syndrome who was referred to our center due to meningitis with Candida albicans.

\section{Case Report}

The patient was a 1 month-old girl, the first child from consanguine parents, who lives in Tehran, capital of Iran. She was preterm infant ( 28 weeks) with very low-birth-weight and cyanosis at birth. She was referred to our center, Children's Medical Center Hospital, at the age of 1 month, with seizure, Received on 10 April 2009; revised 26 October 2009.

Address for correspondence: Dr. Setareh Mamishi.Children's Medical Center Hospital, 62 Qarib St., Keshavarz Blvd., Tehran 14194, Iran. Phone/Fax:+ 98216642 8996. E-mail: smamishi@sina.tums.ac.ir and rezaei_nima@hbi.ir.

The Brazilian Journal of Infectious Diseases

2009;13(6):452-453. (C) 2009 by The Brazilian Journal of Infectious Diseases and Contexto Publishing. All rights reserved. apnea and recurrent vomiting; she was admitted to NICU unit because of recurrent apnea and low consciousness. Phenobarbital 3 mg (IV, BD) and Aminophilin 2 mg (IV, BD) was started for the patient. Despite optimal medical treatment, her condition was unfavorable.

In physical examination, she had wide anterior fontanel $\left(10^{*} 15 \mathrm{~cm}\right.$ ), head circumference (HC) $40 \mathrm{~cm}$, and sunset eye. There was 3/6 soufflé in cardiac examination and mild hepatomegaly in abdominal examination. The results of complete blood count were as follow: white blood cells $(\mathrm{WBC})=10.8$ cells $/ \mathrm{mm}^{3}$, hemoglobin $=12.4 \mathrm{mg} / \mathrm{dL}$, platelets $=$ 292000 cells $/ \mathrm{mm}^{3}$. Sonographic finding showed intaventricular hemorrhage (IVH) grade 3 that was confirmed with computer tomography (CT) scan. Echocardiography revealed atrial septal defect (ASD) and hypertrophic cardiomyopathy (HCM). Chest X-ray demonstrated bilateral fine reticular. Such findings suggested the diagnosis of Noonan syndrome which was consequently confirmed by mutation analysis of the PTPN11 gene.

Clinical findings and CT scan was also indicated hydrocephalous. We designed to introduce recurrent lumbar puncture and external shunt. The results of cerebrospinal fluid (CSF) analysis from lumbar puncture were as follow: glucose $=$ $24 \mathrm{mg} / \mathrm{dL}$, protein $=50 \mathrm{mg} / \mathrm{dL}, \mathrm{WBC}=16$ cells $/ \mathrm{mL}$. Candida albicans was grown in CSF culture as well as blood culture. Amphotrycine B $250 \mu \mathrm{g} / \mathrm{kg}$ (IV) was started for the patient and she responded well to this therapy. One week later, she was transferred to general unit and subsequently discharged, when she was clinically well. She has not experienced any serious complications afterward.

\section{Discussion}

Noonan syndrome is a congenital disorder with several malformations of different organs, mainly craniofacial, skeletal, and cardiac anomalies [1]. The presented case had also several malformations such as wide anterior fontanel, sunset eye, atrial septal defect and hypertrophic cardiomyopathy. 
While aortic coarctation and bicuspid aortic valve are the most common congenital heart anomalies in Noonan syndrome, interatrial septal defect and obstructive or non obstructive hypertrophic cardiomyopathy is also common and can be seen in up to $25 \%$ of the patients. The high incidence of cardiac abnormalities in this syndrome can show the importance of echocardiographic and Doppler evaluation of patients with the Noonan phenotype [5].

Although Candida spp. meningitis is still a rare condition, it is the commonest etiologic agents of fungal neuroinfections [6], which is even more common in the background of immunosuppression [3]. It seems that independent of birth weight, very premature infants, especially who was born at $<26$ weeks, are at a significantly increased risk of systemic infection with Candida albicans [7], which is frequently fatal [8]. Our patient was a preterm infant with some manifestations suggesting meningitis. Candida spp. could involve CNS and cause chronic meningitis that mimics tuberculosis and also Cryptococcus, which is another common fungal meningitides. Special attention has to be paid in the predisposing conditions for the appearance of neurocandidiasis, and neuroimaging techniques and CSF analysis/culture is needed to confirm the diagnosis [9].

Moreover isolation of Candida spp. from normally sterile body fluids in high-risk infants should also be considered pathogenic and therefore appropriate treatment should be started. However, it should be emphasized that normal CSF parameters do not exclude the diagnosis of neonatal candida meningitis and the majority of infants with disseminated candidiasis do not have any positive findings in blood or CSF cultures [10]. So, careful consideration and appropriate treatment is needed in a case of suggestive imaging studies or inflammatory changes in CSF, even if culture of the fluid is negative [4].
Although experience with candida meningitis is limited, early diagnosis and treatment with amphotericin $\mathrm{B}$, and also in combination with 5-fluorocytosine, is effective which the latter offers potential advantages over amphotericin B alone [9]. Meanwhile morbidity and mortality associated with Candida albicans systemic infection is still high, especially in very premature infants [8]. Delayed diagnosis and onset of therapy as well as severe underlying disease can lead to extremely high mortality rate [6].

\section{References}

1. van der Burgt I. Noonan syndrome. Orphanet J Rare Dis 2007;2:4.

2. Sznajer Y., Keren B., Baumann C., et al. The spectrum of cardiac anomalies in Noonan syndrome as a result of mutations in the PTPN11 gene. Pediatrics 2007;119: 1325-31.

3. Sanya E.O., Ameen N.B., Onile B.A. Candida meningitis in a suspected immunosuppressive patient-a case report. West Afr J Med 2006;25:79-81.

4. Faix R.G., Chapman R.L. Central nervous system candidiasis in the high-risk neonate. Semin Perinatol 2003;27:384-92.

5. Loke H.L., Verber I., Szymonowicz W., Yu V.Y. Systemic candidiasis and pneumonia in preterm infants. Aust Paediatr J 1988;24:13842.

6. Njambi S., Huttova M., Kovac M., et al. Fungal neuroinfections: rare disease but unacceptably high mortality. Neuro Endocrinol Lett 2007;28:25-6.

7. Shetty S.S., Harrison L.H., Hajjeh R.A., et al. Determining risk factors for candidemia among newborn infants from populationbased surveillance: Baltimore, Maryland, 1998-2000. Pediatr Infect Dis J 2005;24:601-4.

8. Rodriguez D., Almirante B., Park B.J., et al; Barcelona Candidemia Project Study Group. Candidemia in neonatal intensive care units: Barcelona, Spain. Pediatr Infect Dis J 2006;25:224-9.

9. Sánchez-Portocarrero J., Pérez-Cecilia E., Corral O., et al. The central nervous system and infection by Candida species. Diagn Microbiol Infect Dis 2000;37:169-79.

10. Cohen-Wolkowiez M., Smith P.B., Mangum B., et al. Neonatal Candida meningitis: significance of cerebrospinal fluid parameters and blood cultures. J Perinatol 2007;27:97-100. 